\title{
THE BOLD STRATEGY IN PRESENCE OF HOUSE LIMIT
}

\author{
J. ERNEST WILKINS, JR.
}

\begin{abstract}
It is known that an optimal strategy for a gambler, who wishes to maximize the probability of winning an amount $a-x$ in a subfair red-and-black casino if his initial capital is $x$, is the bold strategy in which the gambler wagers at each opportunity the minimum of his entire current capital $x^{\prime}$ and the amount $a-x^{\prime}$ required to reach the goal $a$ if he wins the bet. If the casino imposes an upper limit $L$ on wagers, we shall prove that the modified bold strategy of wagering $\min \left(x^{\prime}, a-x^{\prime}, L\right)$ is optimal, at least in the important special case in which the goal $a$ is an integral multiple of the house limit $L$.
\end{abstract}

1. The probability of success using the modified bold strategy. Let $P(x)$ be the probability of reaching the goal $a$ from an initial capital $x$ in a redand-black casino [1, Chapter 5], which does not permit wagers exceeding $L$, when the gambler uses the modified bold strategy of wagering $\min \left(x^{\prime}, a-x^{\prime}, L\right)$ when his current capital is $x^{\prime}$. If $p$ is the probability of winning an individual game and $q=1-p$ (so that $0<p<1,0<q<1$ ), it is clear that

$$
P(x)=p P(2 x)
$$$$
0 \leqq x<L
$$

(2) $\quad P(x)=p P(x+L)+q P(x-L)$,

$L \leqq x<a-L$,

$$
P(x)=p+q P(2 x-a),
$$$$
a-L \leqq x<a \text {. }
$$

We assume that $a=n L$ for some integer $n$ greater than 2. (If $n=1$ or 2 , the ordinary bold strategy involves no wagers greater than $L$.)

For each $x$ there exist a unique integer $m$ (the quotient) and a unique number $R$ (the remainder) such that $x=m L+R, 0 \leqq R<L$.

LEMMA 1. The function

$$
P(x)=\frac{[1+(u-1) Q(R / L)] u^{m}-1}{u^{n}-1}, \quad u=q / p,
$$

satisfies equations (1), (2), and (3) if, and only if, the function $Q(f)$ satisfies

Received by the editors March 18, 1971 and, in revised form, July 19, 1971.

AMS 1970 subject classifications. Primary 60J15, 60G99; Secondary 60K10.

Key words and phrases. Gambler's ruin, bold strategy, red-and-black casino. 
the equations

$$
\begin{aligned}
& Q(f)=p Q(2 f), \quad 0 \leqq f<\frac{1}{2}, \\
& Q(f)=p+q Q(2 f-1), \quad \frac{1}{2} \leqq f<1 .
\end{aligned}
$$

We start the proof of this lemma by constructing Table 1 below to record the quotients and remainders of $x, 2 x, x+L, x-L$, and $2 x-a$ in each of six mutually exclusive intervals whose union is the interval $[0, a]$. Using the entries in this table, simple algebraic manipulations suffice to establish the lemma.

TABLE 1. Values of the quotients and remainders for the arguments of $P$ in equations (1), (2), and (3).

\begin{tabular}{lcccc}
\hline \multicolumn{1}{c}{ Interval } & \multicolumn{4}{c}{ Quotient, Remainder } \\
\hline$[0, L / 2)$ & $0, x$ & $2 x$ & $x \pm L$ & $2 x-a$ \\
{$[L / 2, L)$} & $0, x$ & $1,2 x-L$ & & \\
{$[L, a-L)$} & $m, R$ & & $m \pm 1, R$ & \\
{$[a-L, a-L / 2)$} & $n-1, L-a+x$ & & & $n-2,2(L-a+x)$ \\
{$[a-L / 2, a)$} & $n-1, L-a+x$ & & $n-1, L-2 a+2 x$ \\
{$[a, a]$} & $n, 0$ & & & \\
\hline
\end{tabular}

It is known [2] that there is a unique bounded function $Q(f)$ on $[0,1)$ which satisfies equations (5) and (6). In terms of the binary representation of $f, f=\sum_{k=1}^{\infty} f_{k} 2^{-k}, f_{k}=0$ or 1 , this bounded function is

$$
Q(f)=\sum_{k=1}^{\infty} f_{k} p^{k} \prod_{j=1}^{k-1} u^{f_{j}}
$$

If $Q(1)$ is defined as 1 , the function $Q(f)$ is continuous and strictly increasing on $[0,1]$. In addition $Q(f)=f$ if $p=q$, and [3] $Q(f)$ is singular (with respect to Lebesgue measure) if $p \neq q$, and $Q(f)$ satisfies a Hölder condition of order $-\log _{2} \max (p, q)$ on $[0,1]$.

Since the desired solution $P(x)$ of equations (1), (2) and (3) is a probability, it is bounded between 0 and 1 . In view of the probabilistic interpretation [1, p. 85] of $Q(f)$ and the behavior of $m$ and $R$ as functions of $x$, the following theorem is now obvious.

THEOREM 1. The probability $P(x)$ is given by equation (4), in which $Q(R / L)$ is the probability that a gambler with initial capital $R$ will achieve the goal $L$, if he uses the bold strategy. Moreover, $P(0)=0, P(a)=1, P(x)$ is continuous and strictly increasing on $[0, a], P(x)=x / a$ if $p=q$, and $P(x)$ is singular if $p \neq q$.

If $x$ is a multiple of $L$, so that $R=0$, every wager in the modified bold strategy is $L$, and equation (4) reduces to the classical result [4, p. 314] $\left(u^{m}-1\right) /\left(u^{n}-1\right)=1-\left(u^{n}-u^{m}\right)\left(u^{n}-1\right)$. 
2. Optimality of the modified bold strategy. We shall prove the following result.

THEOREM 2. The modified bold strategy is optimal in a subfair $(p<q)$ redand-black casino with house limit, i.e., no strategy has greater probability of success, when the goal $a$ is an integral multiple of the house limit $L$.

In view of [1, Theorem 2.12.1], it is sufficient to show that

$$
P(x)-p P(x+w)-q P(x-w) \geqq 0
$$

when $0 \leqq w \leqq \min (x, a-x, L)$. If $w=L$, then $x \geqq L, a-x \geqq L$ and the lefthand side of the inequality (7) vanishes by virtue of equation (2). Hence we may assume that $w<L$. We tabulate in Table 2 the quotient and remainder for $x+w$ and $x-w$ in each of four mutually exclusive triangles whose union is the square $0 \leqq w<L, 0 \leqq R<L$.

TABLE 2. Values of the quotients and remainders for the arguments $x \pm w$ of $P$ in the inequality (7).

\begin{tabular}{ccc}
\hline & \multicolumn{2}{c}{ Quotient, Remainder } \\
Triangle & $x+w$ & $x-w$ \\
\hline $0 \leqq R<w<L-R$ & $m, R+w$ & $m-1, L+R-w$ \\
$L-w \leqq R<w<L$ & $m+1, R+w-L$ & $m-1, L+R-w$ \\
$L-R \leqq w \leqq R<L$ & $m+1, R+w-L$ & $m, R-w$ \\
$0 \leqq w \leqq R<L-w$ & $m, R+w$ & $m, R-w$ \\
\hline
\end{tabular}

Using the entries in this Table and equation (4), we see that the function $P(x)$ will satisfy the inequality (7) if, and only if, the function $Q(f)$ satisfies the following inequalities:

(8) $Q(f) \geqq p[Q(f+g)+Q(f+1-g)-1]$,

$0 \leqq f<g<1-f$,

(9) $Q(f) \geqq p Q(f+1-g)+q Q(f-1+g), \quad 1-g \leqq f<g<1$,

(10) $Q(f) \geqq p+q[Q(f-g)+Q(f-1+g)], \quad 1-f \leqq g \leqq f<1$,

(11) $Q(f) \geqq p Q(f+g)+q Q(f-g)$,

$0 \leqq g \leqq f<1-g$.

In these inequalities, $f=R / L$ and $g=w / L$.

It is known [1, pp. 87-89] that the inequality (11) holds on the closed triangle $0 \leqq g \leqq f \leqq 1-g$, and (9) is obtained from (11) by replacing $g$ by $1-g$. Moreover, the replacement in (11) of $f$ by $f+\frac{1}{2}$ and of $g$ by $g-\frac{1}{2}$ and $\frac{1}{2}-g$ shows that

(12) $Q\left(f+\frac{1}{2}\right) \geqq p Q(f+g)+q Q(f+1-g)$, $\frac{1}{2} \leqq g \leqq 1-f \leqq 1$,

(13) $Q\left(f+\frac{1}{2}\right) \geqq p Q(f+1-g)+q Q(f+g), \quad 0 \leqq f \leqq g \leqq \frac{1}{2}$. 
Because $q>p$, it follows from (12) if $g \geqq \frac{1}{2}$ and from (13) if $g \leqq \frac{1}{2}$ that

$$
\begin{aligned}
& Q\left(f+\frac{1}{2}\right) \geqq q[Q(f+g)+Q(f+1-g)]+p-q, \\
& 0 \leqq f \leqq g \leqq 1-f .
\end{aligned}
$$

In view of equation (2), $Q\left(f+\frac{1}{2}\right)=p+q Q(2 f)$, and so

$$
Q(2 f) \geqq Q(f+g)+Q(f+1-g)-1, \quad 0 \leqq f \leqq g \leqq 1-f,
$$

whence the inequality (8) follows from equation (1). Similarly, replacement in (11) of $f$ by $f-\frac{1}{2}$ and $g$ by $g-\frac{1}{2}$ and $\frac{1}{2}-g$ shows that

$$
\begin{array}{ll}
Q\left(f-\frac{1}{2}\right) \geqq p Q(f-1+g)+q Q(f-g), & \frac{1}{2} \leqq g \leqq f \leqq 1, \\
Q\left(f-\frac{1}{2}\right) \geqq p Q(f-g)+q Q(f-1+g), & 0 \leqq 1-f \leqq g \leqq \frac{1}{2},
\end{array}
$$

and consequently, since $q>p$,

$$
Q\left(f-\frac{1}{2}\right) \geqq p Q(f-g)+p Q(f-1+g), \quad 1-f \leqq g \leqq f \leqq 1 .
$$

Using equation (1) we see that

$$
Q(2 f-1) \geqq Q(f-g)+Q(f-1+g), \quad 1-f \leqq g \leqq f \leqq 1,
$$

and now the inequality (10) follows from equation (2). This completes the proof of the inequality (7) and hence of Theorem 2.

We can write equation (4) in the form

$$
P(x)=Q(R / L)\left\{\frac{u^{m+1}-1}{u^{n}-1}\right\}+[1-Q(R / L)]\left\{\frac{u^{m}-1}{u^{n}-1}\right\},
$$

and interpret this result as the probability of success using the following strategy. Wager the remainder $R$ boldly in an attempt to achieve the goal $L$. Whether successful or not, continue with constant wagers of $L$. Therefore, the optimal strategy is not unique.

\section{REFERENCES}

1. Lester E. Dubins and Leonard J. Savage, How to gamble if you must. Inequalities for stochastic processes, McGraw-Hill, New York, 1965. MR 38 \#5276.

2. Georges de Rham, Sur certaines équations fonctionnelles, Ecole Polytechnique de L'Université de Lausanne, Centenaire 1853-1953, Ecole Polytechnique, Lausanne, 1953, pp. 95-97. MR 19, 842.

3. R. Salem, On some singular monotonic functions which are strictly increasing, Trans. Amer. Math. Soc. 53 (1943), 427-439. MR 4, 217.

4. William Feller, An introduction to probability theory and its applications, Vol. 1, 2nd ed., Wiley, Ne:v York; Chapman and Hall, London, 1957. MR 19, 466.

Department of Physics, Howard University, Washington, D.C. 20001 